\title{
Discapacidad y su atención por personal sanitario en hospital de tercer nivel en Honduras
}

\author{
Disability and its attention by health personnel in a third level hospital in Honduras \\ José Elpidio Sierra, $\odot^{1}$ Jenny Zelaya, $\odot^{2}$ Andrea Ramírez, $\odot^{3}$ Mauricio Gonzales, $\odot^{4}$ Leonardo Sierra, $\odot^{5}$ \\ Jackeline Alger, ${ }^{6}{ }^{6}$ Edna Maradiaga. $\oplus^{7}$
}

${ }^{1}$ Médico Fisiatra, Posgrado de Rehabilitación, Facultad de Ciencias Médicas (FCM), Universidad Nacional Autónoma de Honduras (UNAH), Tegucigalpa; ${ }^{2}$ Médica Fisiatra, Instituto Hondureño de Seguridad Social (IHSS), Tegucigalpa; ${ }^{3}$ Médica Fisiatra, IHSS, San Pedro Sula; ${ }^{L}$ Licenciado en Informática; Unidad de Investigación Científica (UIC), FCM UNAH, Tegucigalpa; 5 Médico Fisiatra, Posgrado de Rehabilitación, FCM-UNAH, Tegucigalpa; ${ }^{6}$ MD, PhD, UIC FCM UNAH; Departamento de Laboratorio Clínico, Hospital Escuela; Tegucigalpa; ${ }^{7} \mathrm{MD}$, Maestría en Salud Pública, UIC FCM UNAH; Tegucigalpa.

RESUMEN. Antecedentes: El 25\% de hospitalizaciones pueden requerir rehabilitación y en Honduras la discapacidad en hospitales no ha sido considerada. Objetivo: Determinar proporción de discapacidad en pacientes hospitalizados y conocimientos, actitudes y prácticas (CAP) del personal sanitario en discapacidad/rehabilitación, Hospital Escuela, agosto 2016. Métodos: Estudio descriptivo transversal, en pacientes mayores de 18 años hospitalizados aplicándoles cuestionario World Health Organization Disability Assessment Schedule (WHODAS) para identificar discapacidad. Al personal de salud se le aplicó instrumento para identificar CAP de rehabilitación en personas con discapacidad. Se realizó análisis univariado; se obtuvo consentimiento informado escrito. Resultados: De 235 pacientes, 86.8\% (204) tenía discapacidad. De éstos, 68.1\% (139) edad 18-59 años, $53.4 \%$ (109) sexo femenino, $57.8 \%$ (118) discapacidad moderadacompleta. Sólo en 5.4\% (11) se solicitó interconsulta con Servicio de Rehabilitación tras una semana desde ingreso. De 114 participantes del personal sanitario, $50.9 \%$ (58) tenía conocimiento deficiente; enfermeras profesionales $60.9 \%$ (14) y auxiliares de enfermería $75.0 \%$ (30); actitudes fueron positivas excepto reconocer no manejo integral en pacientes $50.9 \%$ (58), médicos especialistas $30.4 \%$ (7) y $32.1 \%$ (9) médicos residentes. De 9 prácticas evaluadas, 4 fueron menos realizadas: postura antiequino, liberación de prominencias óseas $39.5 \%$ (45), respectivamente; manejo de cavidad oral $50.0 \%$ (57), sedestación en cama $50.9 \%$ (58); menos realizadas por médicos residentes $54.6 \%$ y auxiliares de enfermería $58.5 \%$. Discusión: Existe alta proporción de discapacidad con pobre respuesta posiblemente derivada de conocimiento deficiente y baja realización de buenas prácticas en rehabilitación. Debe capacitarse al personal sanitario en reconocer la discapacidad y mejorar trabajo en equipo con Servicio de Rehabilitación. Palabras claves: Clasificación Internacional de Funcionamiento de la Discapacidad y de la Salud, Evaluación de la discapacidad, Honduras, Pacientes internos, Persona con discapacidad.

Recibido: 01-03-2021 Aceptado: 11-06-2021 Primera vez publicado en línea: 26-06-2021 Dirigir correspondencia a: Dr. José Elpidio Sierra

Correo electrónico: jsierra@unah.edu.hn

Declaración de relaciones y actividades financieras y no financieras y conflictos de interés: ninguno.

DOI: https://doi.org/10.5377/rmh.v89i1.11726

(C) 2021 Autor(es): (c) (i)

\section{INTRODUCCIÓN}

El $25 \%$ de los ingresos hospitalarios requieren atención en rehabilitación. ${ }^{1}$ El término rehabilitación se refiere a todas las intervenciones cuyo "objetivo principal es optimizar el funcionamiento y ayudar a las personas con problemas de salud a ser lo más independientes posible y a participar activamente en la sociedad". ${ }^{2}$ Las personas hospitalizadas, por tener un problema de salud, pueden presentar alguna discapacidad cuando interaccionan los problemas de salud (deficiencias) con las limitaciones en actividad y restricción en la participación. ${ }^{3}$ Estas deficiencias pueden ser cualquier enfermedad o lesión que, según estudios, pueden suponer la aparición de discapacidad entre $50-80 \%$ cuando la persona se hospitaliza. ${ }^{4}$

Durante la hospitalización pueden suceder eventos adversos para los cuales el personal de salud debe estar preparado pudiendo pasar por alto su atención siendo esta omisión también un elemento que ocasione discapacidad temporal o incluso permanente en un rango de 2.6-5.2\%., .,6 Estar preparados para eventos adversos supone tener los conocimientos adecuados con el fin de realizar las mejores prácticas. Sin embargo, no es la realidad en algunas instituciones de salud, donde puede no haber protocolos de atención para prevenir discapacidad y por tanto no se realiza intervenciones efectivas como la movilización temprana, y en los hospitales mejor preparados aún se perciben barreras frente a las buenas prácticas en rehabilitación. ${ }^{7,8}$

En Honduras no se cuenta con estudios sobre la situación de las personas hospitalizadas con discapacidad y su atención por el personal de salud. Fundamentados en esto, se diseñó el presente estudio cuyo objetivo fue determinar la proporción de discapacidad en pacientes internados y describir los Conocimientos, Actitudes y Prácticas (CAPs) del personal de salud hacia la discapacidad y rehabilitación en el Hospital Escuela de Tegucigalpa. El estudio se realizó con el fin de contribuir al diseño de estrategias para la prevención y mejorar la atención de las personas que pueden presentar discapacidad relacionada a su internamiento. 


\section{PARTICIPANTES Y MÉTODOS}

Se realizó estudio descriptivo transversal en dos grupos de participantes: a) pacientes mayores de 18 años de edad hospitalizados en salas del Bloque Médico-Quirúrgico, y b) personal de salud en turno A en las salas del mismo bloque, en el Hospital Escuela en agosto de 2016. Se incluyó todo paciente mayor de 18 años de edad hospitalizado y que aceptó voluntariamente la invitación a participar, con posibilidad de brindar información porque no tenía alteraciones del estado de conciencia o contar con un familiar que pudiera brindar información. En el grupo de personal de salud, se incluyó a aquellos que toman decisiones en el cuidado del paciente y con más de un año de experiencia laboral. No hubo criterios de exclusión en ambos grupos.

El tamaño muestral se estimó usando el programa Open Epi en línea y utilizando los parámetros con valor de $p=50 \%$, nivel de confianza del $95 \%$. Se calculó la muestra en el grupo de pacientes cuyo universo fue de 486 en el bloque médicoquirúrgico, resultando en 215 , agregando un $10 \%$ por pérdidas posibles para un total de 235 pacientes. En el grupo de personal de salud, el universo fue de 145 trabajadores en turno $\mathrm{A}$, calculando muestra de 106 trabajadores, agregando $10 \%$ por pérdidas posibles para una muestra final de 116 trabajadores de salud, dos rehusaron participar quedando un total de 114 (Figura 1).

El muestreo en el grupo de pacientes fue por conveniencia en la selección de las salas de internamiento, y una vez seleccionada la sala se tomó un número de sujetos según el porcentaje que cada sala aporta al total de la muestra: De 486 pacientes distribuidos en las salas, se incluyeron siguiendo la ruta de selección los sujetos de estudio de la manera siguiente: En Bloque Médico (Medicina Interna) con seis salas la proporción tomada fue 42\% (99 pacientes), en Bloque Quirúrgico con 4 salas de cirugía general aportando 39\% (92 pacientes), sala de ortopedia con $12 \%$ (28 pacientes), y neurocirugía $7 \%$ (16 pacientes) para un total de 235 sujetos de estudio al levantar la información; se inició con la primera sala escogiendo los pacientes en camas impares (previamente seleccionado) avanzando hasta completar el número de la muestra y así en cada una de

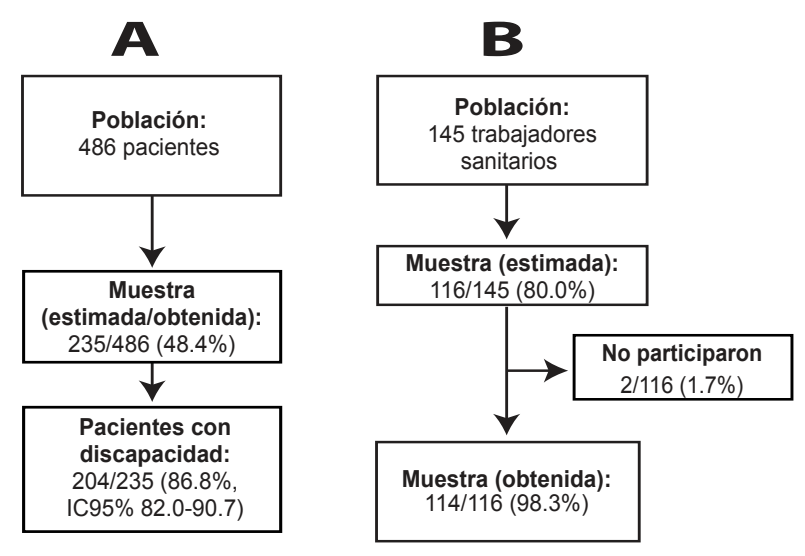

Figura 1. Diagrama de flujo con la descripción del proceso de obtención de las muestras de participantes, Hospital Escuela, agosto 2016. A= Pacientes, B= Trabajadores sanitarios. las salas mencionadas. Para el grupo de personal de salud, el muestreo fue por conveniencia hasta completar la muestra. Del total de personal que trabajaba en turno $\mathrm{A}$ ( 145 trabajadores) en las diferentes salas del Hospital.

Se definió como paciente con discapacidad aquel paciente con pérdida de función y limitación en la actividad o restricción en la participación en los últimos 30 días según el instrumento World Health Organization Disability Assessment Schedule 2.0 (WHODAS). ${ }^{9,10}$ En los pacientes se recolectó información mediante entrevista usando cuestionario diseñado para tal efecto conteniendo datos generales: edad, sexo, ocupación, nivel educativo, procedencia, causa de discapacidad; deficiencias según Clasificación Internacional de Funcionamiento $(\mathrm{CIF})^{3}$ y los dominios del WHODAS 2.0 de doce preguntas versión proxy para identificación de la discapacidad. Estos dominios son cognición, movilidad, actividades de la vida diaria, autocuidado, relaciones interpersonales y participación social. Para grado de severidad, se hizo el análisis del puntaje obtenido usando WHODAS 2.0 en relación con los porcentajes de discapacidad para severidad sugeridos por CIF: sin discapacidad (0-4\%), discapacidad leve (5$24 \%$ ), discapacidad moderada (25-49\%), discapacidad severa (50-95\%), discapacidad completa (96-100\%). También se incluyó preguntas sobre días de hospitalización, si recibió tratamiento de rehabilitación y tiempo en que éste inició. Para el grupo de empleados, se registró información mediante cuestionario autoadministrado conteniendo datos generales: edad, sexo, nivel educativo, rol dentro de sala; se diseñó cuestionario que según la experiencia de los médicos fisiatras puede reunir como mínimo y de manera general CAPs de personal de salud para atención de paciente hospitalizado con discapacidad conteniendo siete preguntas para medir conocimiento sobre rehabilitación, siete preguntas para evaluar actitudes las cuales se midieron usando escala de Likert (1-5) y tres preguntas que evaluaron la realización de buenas prácticas en rehabilitación. Se consideró conocimiento deficiente puntajes $<60 \%$, las actitudes positivas se valoraron por su frecuencia, asimismo la realización de las buenas prácticas. Actitudes neutras se consideraron negativas.

Se diseñó base de datos en Epi Info versión 7.0 (CDC, Atlanta, GA, EUA) en la UIC-FCM-UNAH. Se realizó análisis univariado (frecuencias y porcentajes) con intervalos de confianza del $95 \%$ para la proporción de pacientes con discapacidad y grados de severidad de discapacidad.

El protocolo contó con dictamen del Comité de Ética en Investigación Biomédica (CEIB), FCM UNAH. A cada participante se le explicó el propósito del estudio y se obtuvo consentimiento informado escrito, garantizando la confidencialidad de su información personal. Si el participante no era capaz de comprender la información brindada y responder por sí mismo, un familiar 0 encargado mayor de 18 años tomó la decisión de su participación. Los médicos residentes fueron capacitados mediante el curso en línea de Buenas Prácticas Clínicas (Programa CITI, Universidad de Miami, www.citiprogram.org).

\section{RESULTADOS}

Se encontró 204 sujetos con discapacidad para un porcentaje de $86.8 \%$. (Figura 1) 
Las características sociodemográficas y clínicas de los pacientes y empleados de salud de mayor relevancia fueron las siguientes: De los 204 pacientes con discapacidad, 68.1\% (139) tenía edad entre $18-59$ años, $53.4 \%$ (109) pertenecía al sexo femenino, 91.1\% (168) tenía nivel educativo por debajo del básico o ningún nivel de instrucción, $55.4 \%$ (113) provenía del área rural, $74.5 \%$ (152) estaba desempleado, 53.9\% (110) estaba internado en áreas quirúrgicas, y 50.9\% (104) tenía menos de 7 días de hospitalización. Del personal que trabajaba en el hospital, el $100.0 \%$ tenía edad entre $18-59$ años, $61.4 \%$ (70) pertenecía al sexo masculino, $75.4 \%$ (86) con nivel de instrucción universitaria, y $55.3 \%$ (63) pertenecía al personal de enfermería (Cuadro 1).

De los 204 pacientes con discapacidad, 42.1\% (86) tenía discapacidad leve y $57.8 \%$ (118) tenía discapacidad de moderada a completa. Con respecto a las causas de discapacidad, en $96.6 \%$ (196/203) la causa fue adquirida; en las deficiencias, se encontró que $87.7 \%$ (179) tenían afectadas las funciones sensoriales y dolor, $52.9 \%$ (108) esqueléticas y $45.1 \%$ (92) cardiovascular, mientras que en las estructuras afectadas fueron sistema nervioso central $62.7 \%$ (128), relacionadas con el movimiento $57.4 \%$ (117) y cardiovascular $45.6 \%$ (93) (Cuadro 2). Se encontró que el $5.4 \%$ (11) fueron interconsultados con el servicio de Medicina Física y Rehabilitación, 5 de ellos en los primeros 7-15, 3 entre 16-30 días de hospitalización y 3 en pacientes con más de 30 días de hospitalización, a ninguno de los que tenían

Cuadro 1. Distribución sociodemográfica y clínica de los pacientes y empleados de salud estudiados, Hospital Escuela, agosto 2016.

\begin{tabular}{|c|c|c|}
\hline Principales características & $\begin{array}{c}\text { Pacientes } \\
\mathrm{n}=204 \\
\mathrm{~N}(\%)\end{array}$ & $\begin{array}{c}\text { Personal de } \\
\text { salud } n=114 \\
N(\%)\end{array}$ \\
\hline \multicolumn{3}{|l|}{ Edad (años) } \\
\hline $18-59$ & $139(68.1)$ & $114(100.0)$ \\
\hline$\geq 60$ & $65(31.9)$ & - \\
\hline \multicolumn{3}{|l|}{ Sexo } \\
\hline Masculino & $95(46.6)$ & $70(61.4)$ \\
\hline Femenino & $109(53.4)$ & $44(38.6)$ \\
\hline \multicolumn{3}{|l|}{ Nivel educativo } \\
\hline Ninguna & $36(17.6)$ & - \\
\hline Básico o menor & $150(73.5)$ & $28(24.6)$ \\
\hline Secundario completo & - & - \\
\hline Universitario completo o incompleto & $18(8.8)$ & $86(75.4)$ \\
\hline \multicolumn{3}{|l|}{ Procedencia } \\
\hline Rural & $113(55.4)$ & NA \\
\hline Urbano & $91(44.6)$ & NA \\
\hline \multicolumn{3}{|l|}{ Ocupación } \\
\hline Desempleado & $152(74.5)$ & NA \\
\hline Empleado & $52(25.5)$ & NA \\
\hline Auxiliar de enfermería & NA & $40(35.1)$ \\
\hline Enfermera profesional & NA & $23(20.2)$ \\
\hline Médico Residente & NA & $28(24.5)$ \\
\hline Médico Especialista & NA & $23(20.2)$ \\
\hline \multicolumn{3}{|l|}{ Sala de hospitalización } \\
\hline Quirúrgico & $110(53.9)$ & NA \\
\hline Medicina Interna & $94(46.1)$ & NA \\
\hline \multicolumn{3}{|l|}{ Estancia hospitalaria } \\
\hline 1-7 días & $104(51.0)$ & NA \\
\hline 8-15 días & 65 (31.9) & NA \\
\hline 16-29 días & 27 (13.2) & NA \\
\hline$\geq 30$ días & $8(3.9)$ & NA \\
\hline
\end{tabular}

NA=No aplica discapacidad leve fue solicitada intervención por el servicio de Rehabilitación (Figura 2).

En cuanto a los conocimientos y prácticas en el personal de salud se encontró que el $50.9 \%$ (58) trabajadores de salud encuestados tuvo conocimiento deficiente siendo más frecuente en personal de enfermería. Encontrándose que los grupos más afectados fueron; enfermeras auxiliares en el $75.0 \%$ (30) y enfermeras profesionales en el $60.9 \%$ (14). (Cuadro 3). Las actitudes fueron positivas excepto en el reconocimiento de manejo integral hacia los pacientes en su sala, donde se presentó la frecuencia más baja siendo $50.9 \%$ (58) y ésta se distribuyó de la siguiente manera: médicos especialistas $30.4 \%$ (7), médicos residentes $32.1 \%$ (9), enfermeras profesionales $56.5 \%$ (13). Considerar que el paciente hospitalizado puede tener alguna discapacidad fue la segunda actitud positiva con baja frecuencia siendo $64.9 \%$ (74), donde las enfermeras auxiliares tuvieron la frecuencia más baja con 47.5\% (19). (Cuadro 3 ).

De las nueve buenas prácticas para paciente hospitalizado evaluadas, cuatro fueron las menos frecuentes: postura antiequino $39.5 \%$ (45), liberación de prominencias óseas 39.5\% (45) que tienen que ver con el cuidado de la piel siendo menos puestas en práctica por auxiliares de enfermería; manejo de cavidad oral $50.0 \%$ (57) las cuales son menos indicadas por el personal médico, y sedestación en cama $50.9 \%$ (58) que son menos puestas en práctica por el personal de enfermería. El grupo de profesionales cuya realización de buenas prácticas tuvo la menor proporción fue el de residentes de posgrados médicos con $54.6 \%$ seguido por auxiliares de enfermería con $58.5 \%$ (Cuadro 3).

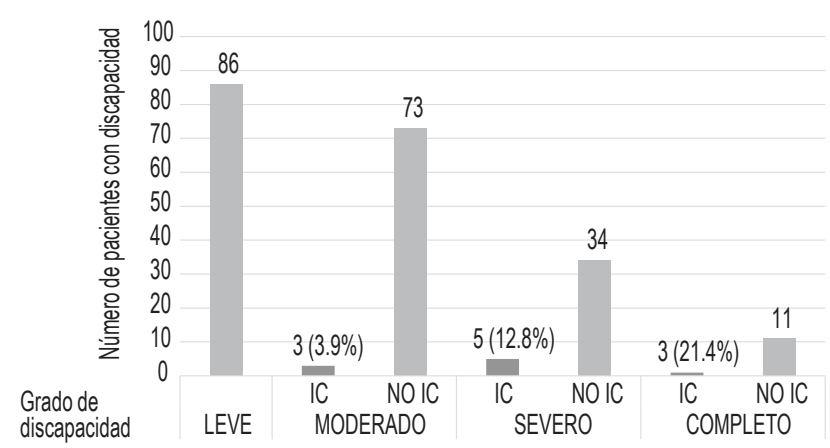

Figura 2. Frecuencia de interconsultas en pacientes con discapacidad según severidad, Hospital Escuela, agosto 2016. IC= Interconsultado a Medicina de Rehabilitación, No IC=No fue interconsultado

\section{DISCUSIÓN}

En este estudio se determinó una alta proporción de discapacidad, el 86.8\% (204) en pacientes internados en un hospital de tercer nivel en Tegucigalpa. Aunque este porcentaje parece elevado, clasificando por severidad $42.2 \%$ (86) presentó una discapacidad leve la cual puede considerarse como temporal sobre todo observando que la mayoría de los pacientes provenía de salas quirúrgicas en las que el dolor posquirúrgico puede limitar la realización de actividades. Sin embargo, más 
Cuadro 2. Distribución de discapacidad por severidad, causa y deficiencias, en pacientes del Hospital Escuela, agosto 2016, $n=204$.

\begin{tabular}{|c|c|c|}
\hline Características & $\mathrm{N}(\%)$ & (IC 95\%) \\
\hline \multicolumn{3}{|l|}{ Severidad de discapacidad } \\
\hline Leve & $86(42.2)$ & $(35.5-49.0)$ \\
\hline Moderada & $73(35.8)$ & $(29.4-42.5)$ \\
\hline Severa & $34(16.7)$ & $(12.0-22.3)$ \\
\hline Completa & $11(5.4)$ & $(2.9-9.2)$ \\
\hline \multicolumn{3}{|l|}{ Causa de discapacidad* } \\
\hline Congénita & $7(3.4)$ & - \\
\hline Adquirida & $196(96.6)$ & - \\
\hline \multicolumn{3}{|l|}{ Función afectada } \\
\hline Sensoriales y de dolor & $179(87.7)$ & - \\
\hline Esqueléticas & $108(52.9)$ & - \\
\hline Cardiovascular & $92(45.1)$ & - \\
\hline Endócrino/metabólicas & $56(27.4)$ & - \\
\hline Neuromuscular & $52(25.5)$ & - \\
\hline Digestivo & $51(25.0)$ & - \\
\hline Genitourinario & $49(24.0)$ & - \\
\hline Piel y relacionadas & $44(21.6)$ & - \\
\hline Respiratorio & $35(17.2)$ & - \\
\hline Mentales & $29(14.2)$ & - \\
\hline Hematológico & $25(12.3)$ & - \\
\hline De la voz y el habla & $23(11.3)$ & - \\
\hline Reproductoras & $21(10.3)$ & - \\
\hline Inmunológico & $12(5.9)$ & - \\
\hline \multicolumn{3}{|l|}{ Estructura afectada } \\
\hline Sistema nervioso & $128(62.7)$ & - \\
\hline Relacionada al movimiento & $117(57.4)$ & - \\
\hline Cardiovascular & $93(45.6)$ & - \\
\hline Metabólico & $53(26.0)$ & - \\
\hline Digestivo & $51(25.0)$ & - \\
\hline Endócrino & $50(24.5)$ & - \\
\hline Genitourinario/Reproductor & $49(24.0)$ & - \\
\hline Piel/relacionados & $42(20.6)$ & - \\
\hline Respiratorio & $32(15.7)$ & - \\
\hline Ojos & $29(14.2)$ & - \\
\hline Relacionados a voz y habla & $25(12.3)$ & - \\
\hline Oídos y relacionadas & $19(9.3)$ & - \\
\hline Inmunológico & $15(7.4)$ & - \\
\hline
\end{tabular}

*No consignado 1

de la mitad de los pacientes encuestados presentó un nivel de severidad moderado a completo necesitando por tanto asistencia para realizar sus actividades (dispositivos y cuidados por terceros) por lo que, sin ayuda, existe riesgo de eventos adversos relacionados con la hospitalización. El porcentaje de discapacidad descrito en otros estudios es variable. Hajjioui y colaboradores en Marruecos encontraron 25.3\% de pacientes con discapacidad de movilidad y $22.1 \%$ en Actividades de Vida Diaria (AVD) que incluyen aseo y vestuario. ${ }^{11}$ Otro estudio en Paraguay ${ }^{12}$ en un hospital especializado en enfermedades oculares en 2018 reportó un porcentaje de $27 \%$ de discapacidad, siendo las más frecuentes en el dominio visual $16.4 \%$, comunicación $9.6 \%$, recordar $5.9 \%$ y movilidad $3.8 \%$. El contraste entre estos hallazgos y los de Marruecos y Paraguay pueden explicarse porque en el primer estudio se utilizó una escala diferente (Escala de Rankin modificada) haciendo que los datos no sean comparables, y en el segundo caso, se utilizó el instrumento del Grupo de Washington que aunque utiliza el lenguaje CIF-OMS, fue aplicado en el contexto de un hospital especializado en enfermedades oculares por tanto la discapacidad en el dominio de visión es la que se refleja con mayor porcentaje.
En la mayoría de los pacientes con discapacidad estudiados se encontró en el grupo de edad entre 18-59 años lo cual es similar lo encontrado por Hajjioui y colaboradores ${ }^{11}$ donde la edad promedio fue de $42.2 \pm 14.2$ en los pacientes cuya discapacidad generaba impacto en el trabajo, lo cual puede explicarse porque el intervalo de edad abarca 41 años (18-59 años) en los cuales es mayor la presencia de enfermedades quirúrgicas y traumáticas siendo una importante causa de internamiento en el hospital estudiado en esta investigación. Se encontró que, entre los pacientes con discapacidad, la mayoría era mujer, con nivel educativo debajo del básico, sin empleo, provenía del área rural, y la causa de la discapacidad fue identificada en más del $90 \%$ como adquirida. Esto concuerda con lo propuesto en el Informe Mundial de la Discapacidad ${ }^{13}$ de la OMS de 2011 el cual describe poblaciones vulnerables que incluye sexo femenino, poca formación académica y sin trabajo o bajos ingresos; además, la discapacidad puede estar influida por las tendencias en los problemas de salud, factores ambientales y otros como accidentes de tráfico, catástrofes naturales, conflictos, hábitos alimenticios y abuso de sustancias.

Las deficiencias de función más frecuentes fueron sensoriales y de dolor, esqueléticas, cardiovasculares y endócrino-metabólico; las deficiencias en estructuras afectadas más frecuentes fueron las de sistema nervioso, relacionadas con movimiento, cardiovascular y metabólico. Estos resultados son parecidos a lo encontrado por Hajjioui y colaboradore ${ }^{11}$ donde en orden de frecuencia fue las deficiencias de dolor, sensorial y de movimiento las más importantes. También en el Informe Mundial de la Discapacidad ${ }^{13}$ se reconoce a las enfermedades crónicas como causa de discapacidad, y, asimismo, Derrett y colaboradores $^{14}$ encontraron que los pacientes hospitalizados pueden tener hasta 3 veces más oportunidad de discapacidad cuando tienen enfermedad crónica que los que no se hospitalizan.

Los conocimientos y prácticas del personal de salud en este nivel de atención parecen ser insuficientes. Aunque la mayoría de las actitudes hacia la rehabilitación son positivas, encontrar que los médicos y enfermeras reconocen carencia de trato integral del paciente a su cargo y a su vez consideran que estos no pueden presentar una discapacidad podría explicar por qué identificamos un porcentaje bajo de solicitud al Servicio de Rehabilitación y se realiza de manera tardía. Alotaibi y colaboradores ${ }^{15}$ realizaron un estudio en personal de salud, educadores y personal sanitario de instituciones privadas en Kuwait donde valoraban el nivel de conocimiento del personal y sus actitudes, encontrando que el puntaje de actitudes tuvo un promedio de 4.4/5 significando actitudes favorables hacia el papel de la terapia ocupacional con puntajes más altos en aquellos profesionales que trabajaban en hospital general o que tenían experiencias de trabajo con terapeutas ocupacionales.

Sobre los conocimientos encontramos que $65.0 \%$ del personal encuestado tenía formación universitaria, y $51.0 \%$ obtuvo puntaje que se puede catalogar como deficiente; tanto en el grupo de médicos como enfermeras, el conocimiento deficiente fue mayor en los grupos con menos años de formación/experiencia profesional como residentes de medicina y auxiliares de enfermería. Calkins y colaboradores ${ }^{16}$ en un estudio realizado 
Cuadro 3. Frecuencia de conocimientos, actitudes y prácticas en el personal de salud, Hospital Escuela, agosto 2016, n=114.

\begin{tabular}{|c|c|c|c|c|c|}
\hline \multirow[b]{2}{*}{ CONOCIMIENTOS/PRÁCTICAS } & \multicolumn{4}{|c|}{ PERSONAL DE SALUD } & \multirow[b]{2}{*}{$\begin{array}{c}\text { TOTAL } \\
\text { n (\%) }\end{array}$} \\
\hline & $\begin{array}{l}\text { Enfermera auxiliar } \\
\qquad \begin{array}{c}n=40 \\
n(\%)\end{array}\end{array}$ & $\begin{array}{c}\text { Enfermera profesional } \\
n=23 \\
n(\%)\end{array}$ & $\begin{array}{c}\text { Médico residente } \\
\mathrm{n}=28 \\
\mathrm{n}(\%) \\
\end{array}$ & $\begin{array}{c}\text { Médico especialista } \\
\mathrm{n}=23 \\
\mathrm{n}(\%)\end{array}$ & \\
\hline \multicolumn{6}{|l|}{ Nivel de conocimiento } \\
\hline Deficiente & $30(75.0)$ & $14(60.9)$ & $8(28.6)$ & $6(26.1)$ & $58(50.9)$ \\
\hline Muy Bueno/Bueno & $10(25.0)$ & $9(39.1)$ & $20(71.4)$ & $17(73.9)$ & $56(49.1)$ \\
\hline \multicolumn{6}{|l|}{ Actitudes (Positivas) } \\
\hline Valoración por fisiatra del paciente & $31(77.5)$ & $22(95.6)$ & $28(100.0)$ & $22(95.6)$ & $103(90.3)$ \\
\hline Paciente beneficiado de rehabilitación & $35(87.5)$ & $22(95.6)$ & $28(100.0)$ & $23(100.0)$ & 108 (94.7) \\
\hline Asignación de fisioterapeuta en sala & $36(90.0)$ & $22(95.6)$ & $25(89.3)$ & $20(86.9)$ & $103(90.3)$ \\
\hline Sala exclusiva de rehabilitación & $32(80.0)$ & $21(91.3)$ & $22(78.6)$ & $16(69.6)$ & $91(79.8)$ \\
\hline Trato integral al paciente en su sala & $29(72.5)$ & $13(56.5)$ & $9(32.1)$ & $7(30.4)$ & $58(50.9)$ \\
\hline Paciente puede mejorar función & $27(67.5)$ & $18(78.3)$ & $23(82.1)$ & $20(86.9)$ & $88(77.2)$ \\
\hline El paciente puede tener alguna discapacidad & $19(47.5)$ & $14(60.9)$ & $21(75.0)$ & $20(86.9)$ & $74(64.9)$ \\
\hline \multirow{2}{*}{\multicolumn{6}{|c|}{ Prácticas realizadas }} \\
\hline & & & & & \\
\hline $\mathrm{Si}$ & $35(87.5)$ & $23(100.0)$ & $27(96.4)$ & $23(100.0)$ & $108(94.7)$ \\
\hline Posturas en cama & & & & & \\
\hline $\mathrm{Si}$ & $32(80.0)$ & $20(87.0)$ & $16(57.1)$ & $15(65.2)$ & $83(72.8)$ \\
\hline Maneja cavidad oral & & & & & \\
\hline $\begin{array}{l}\text { Si } \\
\text { Sedestación en cama }\end{array}$ & $21(52.5)$ & $15(65.2)$ & $11(39.3)$ & $10(43.5)$ & $57(50.0)$ \\
\hline Sedestación en cama & & & & & \\
\hline $\begin{array}{l}\mathrm{Si} \\
\text { Medias antitrombóticas }\end{array}$ & $18(45.0)$ & $10(43.5)$ & $16(57.1)$ & $14(60.9)$ & $58(50.9)$ \\
\hline $\mathrm{Si}$ & $24(60.0)$ & $18(78.3)$ & $24(85.7)$ & $21(91.3)$ & $87(76.3)$ \\
\hline Postura antiequino & & & & & \\
\hline $\mathrm{Si}$ & $13(32.5)$ & $12(52.2)$ & $10(35.7)$ & $10(43.5)$ & $45(39.5)$ \\
\hline Liberar prominencias óseas & & & & & \\
\hline $\begin{array}{l}\text { Si } \\
\text { Hidratar piel }\end{array}$ & $7(17.5)$ & $12(52.2)$ & $11(39.3)$ & $15(65.2)$ & $45(39.5)$ \\
\hline $\begin{array}{l}\mathrm{Si} \\
\text { Cambio postura }\end{array}$ & $29(72.5)$ & $17(73.9)$ & $9(32.1)$ & $15(65.2)$ & $70(61.4)$ \\
\hline $\mathrm{Si}$ & $32(80.0)$ & $20(87.0)$ & $14(50.0)$ & $15(65.2)$ & $81(71.1)$ \\
\hline Promedio de prácticas realizadas & $23.4(58.5)$ & $16.3(70.9)$ & $15.3(54.6)$ & $15.3(66.5)$ & $70.3(61.7)$ \\
\hline
\end{tabular}

en dos hospitales de los Estados Unidos, donde participaron médicos y residentes, encontraron que en general los médicos no reconocieron el $66 \%$ de las discapacidades de sus pacientes, y subestimaron el $28 \%$ de las discapacidades cuando se afectaba un dominio, ascendiendo a $31 \%$ cuando se afectaba más de un dominio de discapacidad, y quienes no reconocieron la discapacidad en los pacientes fueron los residentes atribuyéndose al menor tiempo de experiencia profesional. También Alotaibi y colaboradores ${ }^{15}$ en Kuwait encontraron conocimiento deficiente en personal de salud sobre la importancia de terapia ocupacional en paciente hospitalizado siendo más frecuente en quienes tenían menos experiencia profesional. Los resultados presentes hacen pensar que posiblemente el currículo formativo de las carreras universitarias de la salud no está incorporando temas como discapacidad y rehabilitación contribuyendo a que los egresados desconozcan sobre el tema.

El tener conocimientos sobre discapacidad por debajo del $60 \%$ puede traducirse en prácticas menos efectivas para evitar la aparición de discapacidad relacionada con la enfermedad y complicaciones, tal como muestran estos resultados donde en 4 de las 9 buenas prácticas en paciente encamado la frecuencia de realización no sobrepasa el $50 \%$. Los grupos con menor frecuencia de prácticas fue auxiliares de enfermería (58.5\%) y mé- dicos residentes (54.6\%). Las prácticas realizadas con mayor frecuencia fueron la solicitud de interconsulta, las relacionadas con el cuidado de piel y uso de medias antitrombóticas, dejando con menos frecuencia de realización algunas que tienen que ver con la movilización temprana y profilaxis de broncoaspiración en pacientes con disfagia. Qutub y colaboradores ${ }^{17}$ mediante encuesta que valora el conocimiento sobre movilización temprana en cuidado intensivo en terapistas respiratorios de Arabia Saudita, encontraron que el puntaje de conocimiento sobre el tema era de $62.8 \pm 21.3$ (bueno); sin embargo, la realización de la movilización temprana en los pacientes fue de $42 \%$ de los encuestados, y solo el $16 \%$ de ellos iniciaron la movilización tan temprano como a las 24 horas post extubación. Muchas de las prácticas no se realizan por el desconocimiento de la seguridad de las mismas como en lo reportado por Brown y colaboradores ${ }^{18}$ quienes encontraron que las barreras percibidas tanto por pacientes como por personal sanitario para movilizar los pacientes tempranamente fueron miedo a las caídas, presencia de catéter venoso o vía intravenosa, falta de motivación y debilidad en los pacientes. No realizar movilización temprana en los pacientes puede relacionarse con desacondicionamiento y baja funcionalidad como evidencia la publicación de Kirk y colaboradores ${ }^{19}$ quienes han observado que por cada hospitali- 
zación de corta duración hay un aumento en la dependencia en Actividades de la Vida Diaria (AVD) al egreso, y niveles bajos de movilidad durante el internamiento ha sido percibido por los pacientes como un riesgo alto de disminución funcional.

En relación con el manejo de cavidad oral que consiste en el tratamiento para disfagia sobre todo en pacientes con ictus, fue una de las prácticas menos realizadas $(50 \%$ de práctica) siendo menos frecuente en el grupo de médicos, y es una práctica que reduce el riesgo de neumonía asociada al ictus (aspiración), Eltringham y colaboradores ${ }^{20}$ encontraron en una revisión sistemática evidencia creciente que el diagnóstico y manejo temprano reduce la oportunidad de neumonía asociado a ictus. Por último, los eventos que puedan pasar durante una hospitalización y el manejo adecuado e integral pueden incidir en la funcionalidad sobre todo en pacientes ancianos, tal como encontró Gill y colaboradores ${ }^{21}$ en estudio de población en Estados Unidos evaluando el impacto de cada hospitalización en el último año de vida, encontrando que "las enfermedades agudas y lesiones que provocan hospitalización inciden en la discapacidad en el último año de vida".

Según estos resultados se puede considerar que no se está resolviendo la situación de discapacidad de los pacientes de manera adecuada y oportuna, ya que solo el $5.4 \%$ de los pacientes identificados con discapacidad fueron interconsultados con el Servicio de Rehabilitación, la mitad de ellos fue solicitada tras una semana desde su ingreso, y la mitad restante a dos semanas desde su ingreso. Estos datos son parecidos a los encontrados en el estudio de Hoffman y colaboradores en Chile ${ }^{22}$ donde del total de pacientes que ameritaban rehabilitación, solo $4.3 \%$ de todos los pacientes recibió evaluación por el Médico Especialista en Rehabilitación aumentando este porcentaje a $7 \%$ en quienes tenían una deficiencia que requiere rehabilitación, y a $14 \%$ cuando se trataba de manejo por equipo interdisciplinario de rehabilitación con o sin participación de fisiatra. Esto sugiere ausencia de protocolos dentro de la institución y del personal de salud para poder identificar el canal de atención adecuada para las personas con discapacidad que requieren rehabilitación constituyendo esto un obstáculo discapacitante como reconoce la OMS en el Informe Mundial sobre la Discapacidad ${ }^{13}$ llamándolo problemas en la prestación de servicios. Tan y colaboradores ${ }^{23}$ estudiaron 448 pacientes hospitalizados por el Servicio de Medicina Interna en un hospital de Singapur encontrando correlación entre un puntaje bajo en la Medida de Independencia Funcional (MIF), sobre todo en funciones motoras, con la estancia hospitalaria prolongada, por tanto, recomendaron realizar programas de rehabilitación a los pacientes con alteraciones funcionales para acortar estancia hospitalaria y prevenir re-hospitalizaciones.

Este estudio tiene como limitante no haber aleatorizado la muestra calculada pues las salas (unidades de muestreo) fueron seleccionadas por conveniencia de manera que no podemos generalizar los resultados. La selección fue así para asegurar que con la participación de todas las salas no hubiese sobrestimación de la discapacidad ya que algunas como ortopedia o neurocirugía por las patologías que en ellas se tratan tienden a presentar porcentaje de discapacidad mayor que otras; consideramos necesario realizar un estudio similar en el resto de los hospitales de tercer nivel del país. Al ser estudio descriptivo no hemos utilizado estadística inferencial que nos permita tomar decisiones, por lo que los futuros estudios que sobre el tema puedan desarrollarse podrían incluir análisis que permita inferencia estadística. El cuestionario sobre CAPs fue construido en base a lo que se encuentra en literatura sobre discapacidad y rehabilitación tanto en conocimiento mínimo para el personal de salud, actitudes adecuadas y buenas prácticas en paciente hospitalizado siendo una oportunidad de crear y validar un instrumento para tal fin.

En conclusión, existe alta proporción de discapacidad en los pacientes del área médico-quirúrgica del Hospital Escuela y más de la mitad de ellos con severidad moderada a completa necesitando asistencia para movilizarse o realizar acciones de autocuidado. Aun así, la respuesta por el personal parece ser deficiente, posiblemente por desconocimiento en prevención y manejo del encamamiento, prácticas no realizadas a los pacientes a pesar de que la mayoría muestra buenas actitudes hacia los mismos. Es evidente la necesidad de sugerir a las autoridades de salud fortalecer el Departamento de Rehabilitación existente, para que dirija la política de atención en discapacidad y rehabilitación no solo en lo asistencial, sino en capacitar al personal de salud de las diferentes salas de internamiento en el tema de discapacidad, su reconocimiento, prevención, y realización de buenas prácticas en rehabilitación para beneficio de los pacientes.

\section{CONTRIBUCIONES}

Todos los autores participaron en la concepción y el diseño del estudio. JZ y AR recolectaron la información. JES y MG lideraron el desarrollo de la base de datos y JES lidero el análisis de los resultados. JES lideró la redacción del artículo. Todos los autores participaron de la revisión del artículo en el proceso editorial y aprobaron la versión final del mismo.

\section{AGRADECIMIENTOS}

Se reconoce y agradece a la Unidad de Investigación Científica (UIC), Facultad de Ciencias Médicas UNAH por la gestión que permitió la participación de los autores JES, JA, EM, MG en el Taller sobre Escritura de Artículos Científicos, desarrollado del 30 de octubre al 1ro de noviembre de 2019 en Tegucigalpa, bajo la coordinación de las Dras. Maria Elena Peñaranda y Maria Patricia Arbelaez, Sustainable Sciences Institute (SSI), San Francisco, California, Estados Unidos de América.

\section{DETALLES DE LOS AUTORES}

${ }^{1}$ José Elpidio Sierra

2Jenny Zelaya

${ }^{3}$ Andrea Ramírez

4Mauricio Gonzales

${ }^{5}$ Leonardo Sierra

${ }^{6}$ Jackeline Alger

${ }^{7}$ Edna Janeth Maradiaga

jsierra@unah.edu.hn zelayajenny14@gmail.com acra_21@yahoo.com gonzalesmauricio721@gamil.com faraonramsesii@yahoo.com jackelinealger@gmail.com edjamar3006@yahoo.com

${ }^{2,3}$ Residente del Posgrado de Rehabilitación, Facultad de Ciencias Médicas, Universidad Nacional Autónoma de Honduras (UNAH), Tegucigalpa, Cohorte 2014-2016. 
1. Agencia de Cooperación del Japón. Estudio de prevalencia de discapacidad en la región oriental del Paraguay: informe final. Asunción: Delta Consultora Integral Asunción; 2012.

2. Gimigliano F, Negrini S. The World Health Organization. Rehabilitación 2030: a call for action. Eur J Phys Rehabil Med. [Internet]. 2017 [citado 25 febrero 2021]; 23(2):155-68. Disponible en: The World Health Organization "Rehabilitation 2030: a call for action" - European Journal of Physical and Rehabilitation Medicine 2017;53(2):155-68 - Minerva Medica - Journals

3. World Health Organization. International classification of functioning disability and health. Geneva: WHO; 2001.

4. Gill TM, Allore HG, Holford TH, Guo Z. Hospitalization, restricted activity, and development of disability among older persons. JAMA [Internet]. 2004 [citado 24 febrero 2021];292(17):2115-24. Disponible en: https://pubmed. ncbi.nlm.nih.gov/15523072/

5. Baker GR, Norton PG, Flintoft V, Blais R, Brown A, Cox J, et al. The Canadian adverse event study: the incidence of adverse events among hospital patients in Canada. CAMJ [Internet]. 2004 [citado 24 febrero 2021];170(11):1678-86. Disponible en: https://pubmed.ncbi.nlm.nih. gov/15159366/

6. Brennan TA, Leape LL, Laird NM, Hebert L, Localio R, Lawthers AG, et al. Incidence of adverse events and negligence in hospitalized patients. $\mathrm{N}$ Engl J Med [Internet]. 1991 [citado 24 febrero 2021];324(6):370-76. Disponible en: https://pubmed.ncbi.nlm.nih.gov/1987460/

7. Kim C, Kim S, Yang J, Choi M. Nurses' perceived barriers and educational needs for early mobilization of critical ill patients. Aust Crit Care [ Internet]. 2019 [2021 Feb 24]; 32(6): 451-57. Disponibe en: https://pubmed.ncbi.nlm. nih.gov/30591311/

8. Fontela PC, Forgiarini LA, Friedman G. Clinical attitudes and perceived barriers to early mobilizations of critically il patients in adult intensive care unit. Rev Bras Ter Intensiva. 2018:30(2):187-94.

9. Ustun TB, Kostanjesek N, Chatterii S, Rehm J, World Heatlh Organization. Measuring Health and Disability, Manual for WHO Disability Assessment Schedule WHODAS 2.0. Malta: WHO; 2010

10. Federici S, Bracalenti M, Meloni F, Luciano JV. World Health Organization disability assessment Schedule 2.0: An international systematic review. Disabil Rehabilit [Internet]. 2017 [citado 25 febrero 2021];39(23):2347-80.

11. Hajjioui A, Fourtassi M, Nejjari C. Prevalence of disability and rehabilitation needs amongst adult hospitalized patients in a Moroccan university hospital. J Rehabil Med. 2015;47(7):593-98.

12. Mörchen M, Zambrano O, Paez A, Salgado P, Peniecook J, Brandt von Lindau A, et al. Disability-disaggregated data collection: hospital-based application of the Washington Group Questions in an Eye Hospital in Paraguay. Int J Environ Res. Public Health. [Internet]. 2019 [citado 25 febrero 2021];16(17):16. Disponible en: https://www.ncbi.nlm.nih.gov/pmc/articles/ PMC6747208/

13. World Health Organization. Wolrd Report on Disability. Malta: WHO; 2011.

14. Derrett S, Wilson S, Samaranayaka A, Langley J, Wyeth E, Ameratunga $S$, et al. Prevalence and predictors of disability 24-months after injury for hospitalised and non-hospitalised participants: results from a longitudinal cohort study in New Zealand. PloS one. [Internet]. 2013 [citado 24 febrero 2021];8(11):14. Disponible en: https://journals.plos.org/plosone/ article?id=10.1371/journal. pone. 0080194

15. Alotaibi NM, Manee FS, Murphy LJ, Rassafiani M. Knowledge about and attitudes of interdisciplinary team members toward occupational therapy practice: implications and future directions. Med Princ Pract. 2019; 28:15866.

16. Calkins DR, Rubenstein LV, Cleary PD, Davies AR, Jette AM, Fink A, et al. Failure of physicians to recognize functional disability in ambulatory patients. Ann Intl Med. 1991;114:451-54.

17. Qutub HO, Matani AS, Faroogi FA. Survey of respiratory care professionals' knowledge in early mobilization: A pilot study. J Epidemiol Glob Health. 2018;8(3-4):208-12.
18. Brown CJ, Williams BR, Woodby LL, Davis LL, Allman RR. Barriers to mobility during hospitalization from the perspectives of older patients and their nurses and physicians. J Hosp Med. 2007;2:305-13.

19. Kirk JW, Bodilsen AC, Thomsen TT, Pedersen MM, Bandholm T, Husted RS, et al. A tailored strategy for designing the Walk-Copenhagen (WALK$\mathrm{Cph}$ ) intervention to increase mobility in hospitalised older medical patients: a protocol for the qualitative part of the WALK-Cph Project. BMJ Open. [Internet]. 2018 [citado 24 febrero 2021];8:e020272. Disponible en: https://bmjopen.bmj.com/content/bmjopen/8/3/e020272.full.pdf

20. Eltringham SA, Kilner K, Gee M, Sage K, Bray BD, Pownall S, et al. Impact of dysphagia assessment and management on risk of stroke-associated pneumonia: A systematic review. Cerebrovasc dis. 2018; 46:97-105.

21. Gill TM. Gahbauer EA, Han L, Allore HG. The role of intervening hospital admissions on trajectories of disability in the last year of life: prospective cohort study of older people. BMJ. [Internet]. 2015 [citado 25 febrero 2021];350:h2361 Disponible en: https://www.ncbi.nlm.nih.gov/pmc/articles/PMC4443433/ doi: 10.1136/bmj.h2361.

22. Hofmann N, Paolinelli C. Castro A. Características de la población y atenciones en rehabilitación que recibe en hospital general. Rev Med Chile. 2017; 145:1541-50.

23. Tan C, Sien Y, Koh GC, De Silva D, Earnest A, Barbier S. Disability impacts length of stay in general internal medicine patients. J Gen Intern Med. 2014;29(6):885-90.

ABSTRACT. Background: In hospitalizations, 25\% may require rehabilitation and in Honduras disability in hospitals has not been considered. Objective: To determine the proportion of disability in hospitalized patients and Knowledge, Attitudes and Practices (KAP) of health personnel towards disability/rehabilitation, Hospital Escuela, August 2016. Methods: Descriptive cross-sectional study, in hospitalized patients older than 18 years of age applying World Health Organization Disability Assessment Schedule (WHODAS) questionnaire to identify disability. A questionnaire was applied to the health personnel to identify rehabilitation KAP in people with disabilities. Univariate analysis was performed, written informed consent was obtained. Results: From 235 patients, 86.8\% (204) had disability. From these, $68.1 \%(139)$ age $18-59$ years, $53.4 \%$ (109) female, $57.8 \%$ (118) moderate-complete disability. Only $5.4 \%$ (11) were consulted with the Rehabilitation Service after one week of admission. From 114 health personnel participants, 50.9\% (58) had poor knowledge, professional nurses $60.9 \%$ (14) and nursing assistants $75.0 \%$ (30); Attitudes were positive except recognizing non-comprehensive management in patients $50.9 \%$ (58), specialist physicians $30.4 \%$ (7) and $32.1 \%$ (9) resident physicians. From 9 practices evaluated, 4 were less performed: anti-equine posture, release of bony prominences $39.5 \%$ (45) respectively; oral cavity management $50.0 \%$ (57) and sitting in bed $50.9 \%$ (58), less performed by resident physicians $54.6 \%$ and nursing assistants $58.5 \%$. Discussion: There is a high proportion of disability with poor response possibly derived from poor knowledge and low performance of good practices in rehabilitation. Health personnel should be trained to recognize disability and improve teamwork with the Rehabilitation Service.

Keywords: Disability and Health Inpatient, Disability evaluation, Disabled person, Honduras, International Classification of Functioning, Disability and Health. 